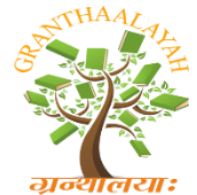

\author{
INTERNATIONAL JOURNAL OF RE
GRANTHAALAYAH \\ A knowledge Repository
}

Science

\title{
DEMONSTRATION OF ELECTROMAGNETIC RADIATION FROM MAGNETITE LIKE RESPIRABLE AIR POLLUTION PARTICULATES SUPPORTING EPIDEMIOLOGICAL FINDINGS IN CANCER GENESIS
}

\author{
Abrahám A. Embí BS MBA *1 \\ *1 13442 SW 102 Lane, Miami, Florida, 33186 USA
}

\begin{abstract}
The purpose of this manuscript is to expand on previous findings where an abrupt change in light electromagnetic spectrum was noticed during blood coagulation. In vitro experiments are introduced showing an electromagnetic radiation effect expressed as color changes observed on fresh blood smears undergoing water evaporation. Foreign particles, such as magnetized magnetite fragments and paramagnetic iron filings were placed on a fresh blood smear and then monitor and recorded by video-microscopy. The recent introduction of an in vitro blood smear technique allowing for the temporary preservation of fresh blood properties has allowed for this discovery. Recently, a promising use of magnetite as life saving tool by controlling internal bleeding was proposed; in vitro studies confirmed a decrease in hemostasis time in a blood vessel by a factor of 6.5. Magnetite is described as an iron oxide that occurs naturally on Earth, and has been detected in the form or nanoparticles in the human brain; which is attributed to biogenic (produced by living organisms) or anthropological particles (environmental pollutants) causes. Additionally, magnetite was researched and found to be non-toxic; to the point of obtaining having FDA approval to be used as a contrast media infusion in the blood stream to improve diagnostic procedures, such as in Magnetic Resonance Imaging (MRI).

Materials and Methods

Rock magnetite fragments of different sizes and iron metal filings. Glass slides $(25 \times 75 \times 1 \mathrm{~mm})$. Video microscope Celestron Model \# 44348. Images obtained and digitally downloaded to and Apple MacBook Pro Photo Application. Different in vitro techniques were used for this research. Results

Documented were human blood tissue attracted and adhering to foreign particles (Magnetite and Iron filings) when in direct contact with fresh human blood on fresh glass smears. Also documented were changes in the visible light radiation spectrum manifested as color changes.

\section{Conclusions}

Energy changes were documented during the human blood coagulation cascade. In addition, bioactive magnetite fragments triggered blood clots when placed in direct contact with fresh blood; iron filings also had a similar blood clotting effect. During the coagulation cascade both the iron filings and magnetite were documented inducing a notable shift in the visible light radiation spectrum, thus implying energy changes. The magnetic fields of magnetite induced changes in the form an aura of dark to light pink color, surrounded by a purple/blue hue; and consistent with a biophysical phenomenon. The iron filings also induced fluctuations in light radiation when alone
\end{abstract}


or within the magnetic reach of magnetite, thus confirming magnetite induced magnetic fields also as a trigger for energy fluctuations during blood coagulation. The herein introduced findings are in support of "One additional cause of the origin of cancer is directly related to exogenous and/or endogenous EMFs damaging the DNA within the eukaryotic cell”.

Keywords: Magnetite Fragments; Interfacial Adsorption; Temporary; Fresh Blood Smears; Blood Clots Formation; Magnetite Blood Interactions; Iron Filings Blood Interactions; Electromagnetic Fields and Diseases.

Cite This Article: Abrahám A. Embí. (2019). "DEMONSTRATION OF ELECTROMAGNETIC RADIATION FROM MAGNETITE LIKE RESPIRABLE AIR POLLUTION PARTICULATES SUPPORTING EPIDEMIOLOGICAL FINDINGS IN CANCER GENESIS." International Journal of Research - Granthaalayah, 7(10), 280-292. 10.29121/granthaalayah.v7.i10.2019.398.

\section{Introduction}

The purpose of this manuscript is to expand on previous findings where an abrupt change in the visual light electromagnetic spectrum was noticed during blood coagulation; and at the time attributed to light intensity changes due to water evaporation (Fig.1) (1,2). In vitro experiments are now introduced showing the effects of visible light electromagnetic spectral changes expressed as different colors observed on fresh blood smears undergoing water evaporation (coagulation). Foreign particles, such as magnetized magnetite fragments and paramagnetic iron filings were placed on fresh smear, monitored and recorded by video-microscopy. The recent introduction of an in vitro blood smear technique allowing for the temporary preservation of fresh blood properties; and allowed for this discovery (3). Recently, a promising use of magnetite as lifesaving tool by controlling internal bleeding was proposed; in vitro studies confirmed a decrease in hemostasis time (coagulation time) in a blood vessel (4). Magnetite is described as an iron oxide that occurs naturally on Earth, and has been detected in the form or nanoparticles in the human brain; which is attributed to biogenic (produced by living organisms) or anthropological particles (environmental pollutants) causes (5). Additionally, magnetite was researched and found to be non-toxic; to the point of obtaining having FDA approval to be used as a contrast media infusion in the blood stream to improve diagnostic procedures, such as in Magnetic Resonance Imaging (MRI).

\section{Materials and Methods}

Metal iron filings purchased online and magnetite rocks from Costa Rica were obtained. Small fragments of magnetite similar in size to the iron filings were obtained by rubbing the magnetite rock onto a white $1 \times 2$ " porcelain streak plate. The iron particles were carefully harvested from the container by a wooden toothpick and sprinkled onto a slide. The magnetite particles transferred onto a glass slide by scraping or tapping the streak plate. Fresh whole blood was milked after small needle puncture of the author's hand distal digit and smeared in a $25 \times 75 \times 1 \mathrm{~mm}$ glass slide. 


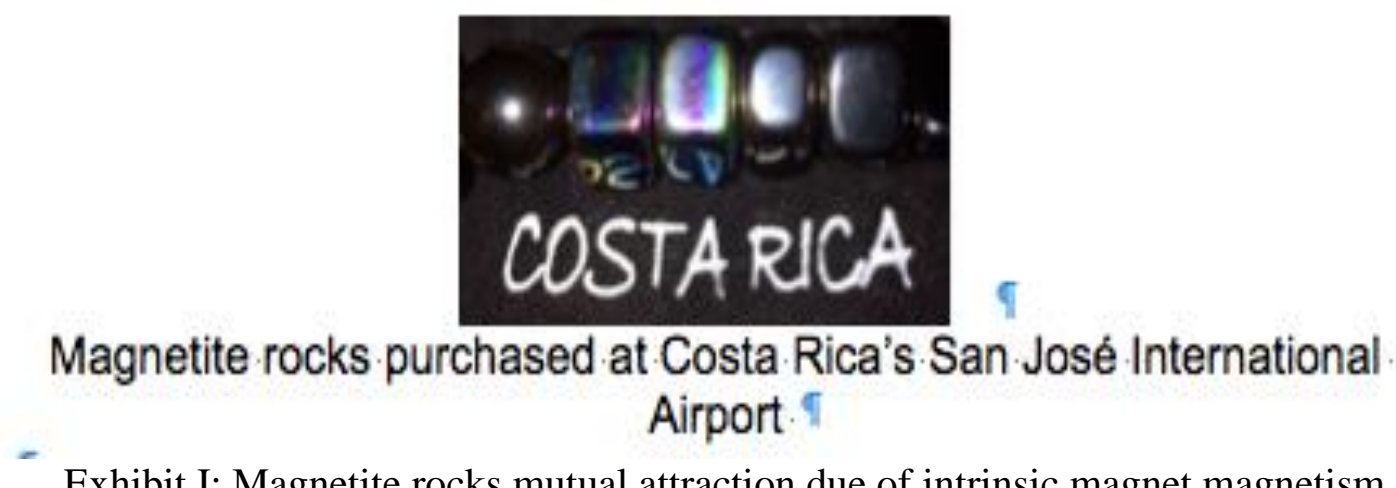

Exhibit I: Magnetite rocks mutual attraction due of intrinsic magnet magnetism

Several in Vitro Techniques Were Used for This Research

1) Methodology to Document Light Radiation Fluctuations during Water Evaporation (Read Coagulation) in Fresh Blood Smears.

Procedure: A fresh blood smear was prepared and continuously video recorded.

2) Methodology to Document and Contrast Fresh Blood Interaction Between Permanently Magnetized Particles (Magnetite) and Non-Magnetized Iron Filings Particles Placed of Fresh Blood Smears.

\section{Procedures}

- Metal iron filings were scattered on fresh blood smears and continuously video recorded until the end of the coagulation cascade or cycle.

- Small magnetite fragments (approximately the size or iron filings) were also placed on fresh blood smears and continuously video recorded until the end of the coagulation cascade or cycle.

To photograph the underside or fragments: After a period of one hour, both A and B preparations were placed on the microscope and the foreign substances (metal filings and magnetite) fragments were physically dislodged via a wooden toothpick.

3) Methodology: to Measure the Magnetic Reach of Hematite on Adjacent Metal Iron Filings Placed on a Glass Slide as Sentinels.

\section{Procedure}

- A $1 \mathrm{~cm}$ long irregular magnetite fragment was sandwiched between two 25x75x1mm glass slides. On the top slide, iron particles were sprinkled within the magnetic reach of the magnetite. The magnetic reach was determined when iron filings were seen attracted by the magnetite (approximately $1 \mathrm{~cm}$ ). In a clean slide (top of sandwiched) a blood smear was then prepared, and iron particles smeared, taking into account the underlying hematite magnetic reach. 
4) Methodology: To Document The Lateral (Same Plane) and Vertical (Through A 1 Mm Thick Glass) Magnetic Effect of Magnetite on Iron Particles in Fresh Blood Undergoing Coagulation.

\section{Procedures}

- Vertical effect: A $1 \mathrm{~cm}$ long irregular magnetite fragment was sandwiched between two 1 mm thick glass slide. On the top slide iron filings were scattered on a fresh blood smear within $1 \mathrm{~cm}$ of the Magnetite. Video recorded during water evaporation.

- Lateral Effect: On a fresh blood smear a $1 \mathrm{~cm}$ long magnetite fragment was placed at the left end of the slide. Iron filings sprinkled on the magnetic reach area $(1 \mathrm{~cm} \pm 3 \mathrm{~mm})$. Blood smear allowed to evaporate and video recorded.

\section{Equipment Used}

Video microscope Celestron Model \# 44348, 25x75x1 mm clean glass slides. Images obtained and then digitally downloaded to and Apple MacBook Pro Photo Application.

\section{Results}

1) Methodology to Document Light Radiation Fluctuations during Water Evaporation (Read Coagulation) in Fresh Blood Smears.

When a blood smear was allowed to undergo unimpeded evaporation on a glass slide, there were observed monochromatic (white) light radiation fluctuations (1) (Fig 1).

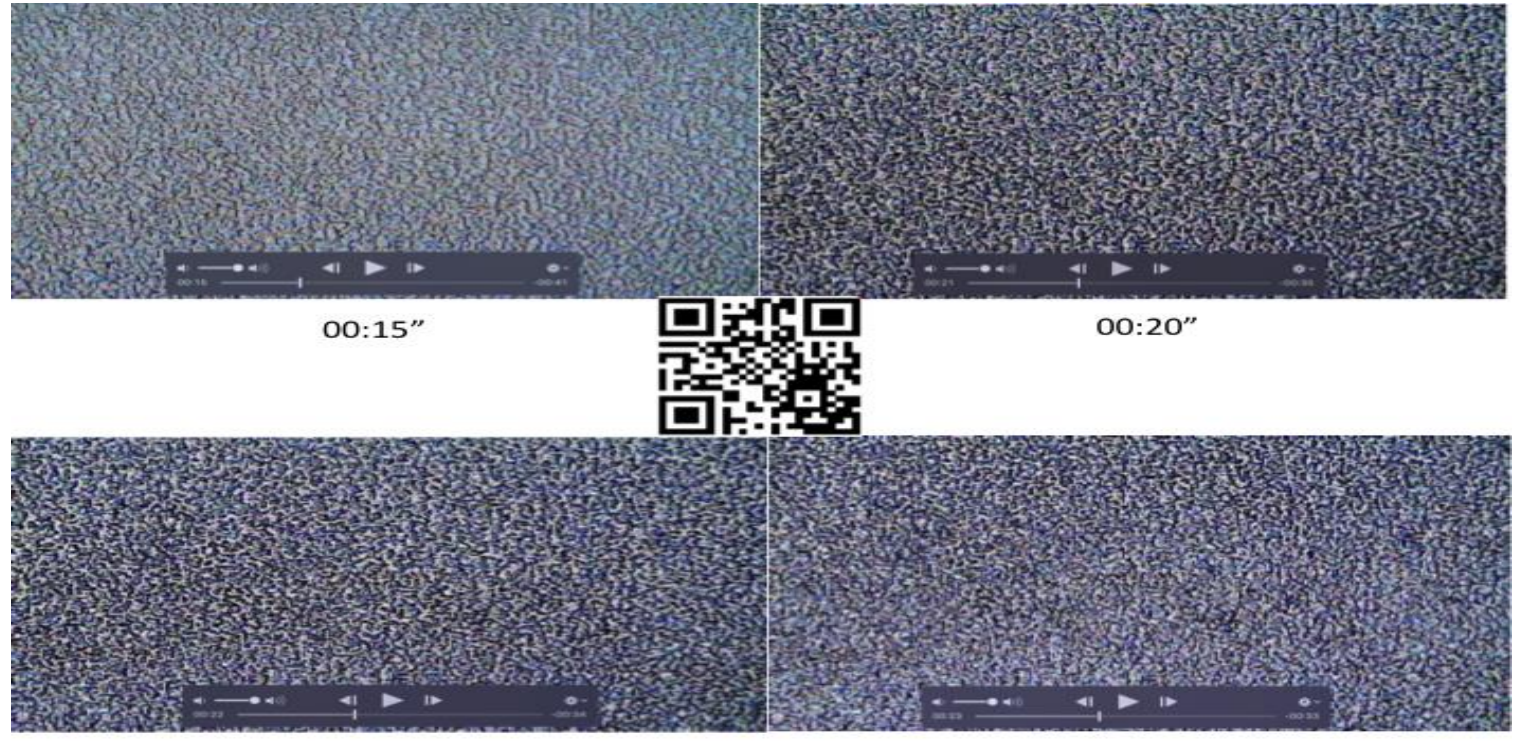

$00: 22 "$

$00: 23 "$

Figure 1: Selected video frames from fresh blood smear allowing for unimpeded evaporation.

Notice the light radiation fluctuations detected by optical microscopy throughout the water evaporation cycle during coagulation cascade. For additional details, please link to: https://youtu.be/MQ3HnQQORgc

Or visit QR Code in image center. 
2) Methodology to Document and Contrast Fresh Blood Interaction Between Permanently Magnetized Particles (Magnetite) and Non-Magnetized Iron Filings Particles Placed of Fresh Blood Smears.

\section{Non-Magnetized Iron Filings}

When iron filings were placed on fresh blood, a symmetrical attraction towards the iron was documented (Fig 2)

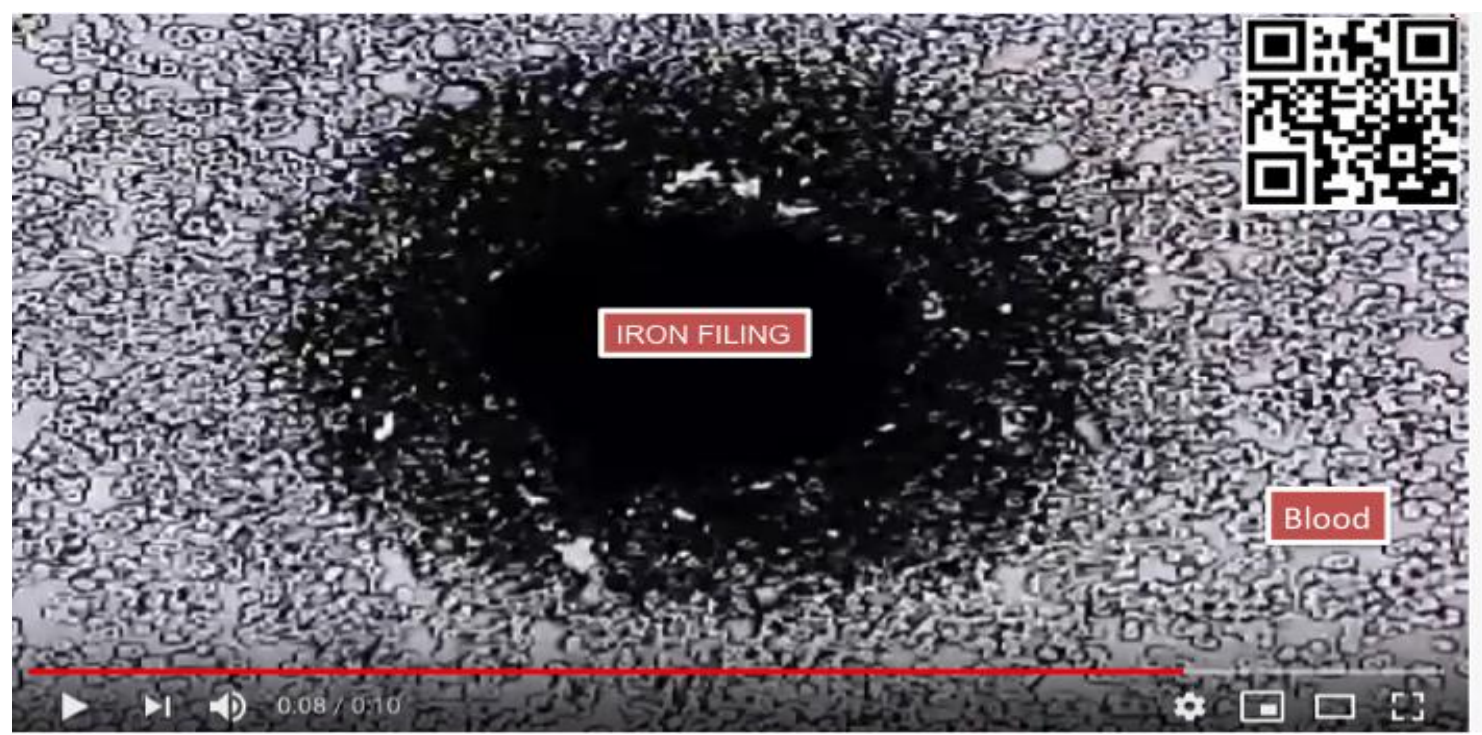

Figure 2: Iron filing in fresh blood smear attracting adjacent blood. For additional details link to: https://youtu.be/hy1Of-wAcS4 Or Scan QR Code in right upper side of image.

Upon removal of the iron particle, deformed RBCs and blood clots noticed (Fig 2A).

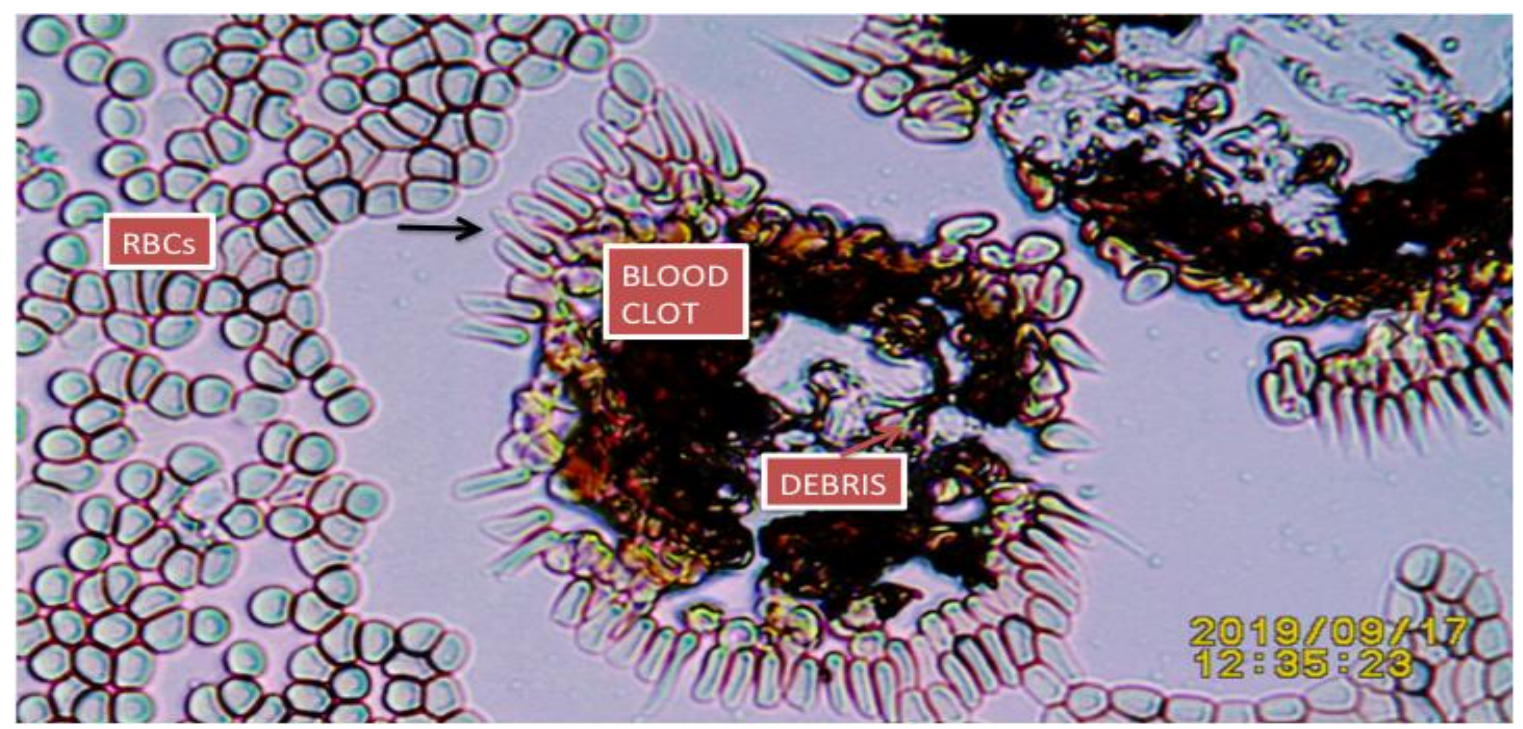

Figure 2A: Microphotograph of video frame after evaporation of metal iron filings placed on fresh smear.

Back Arrow= Deformed RBCs. Blood clot and cellular debris. 
3) Methodology: to Measure The Magnetic Reach of Hematite on Adjacent Metal Iron Filings Placed on A Glass Slide As Sentinels.

\section{No Images Shown}

A $1 \mathrm{~cm}$ long irregular magnetite fragment was sandwiched between two 25x75x1mm glass slides. On the top slide, iron particles were sprinkled within the magnetic reach of the magnetite. The magnetic reach was determined when iron filings were seen attracted by the magnetite (approximately $1 \mathrm{~cm}$ ). In a clean slide (top of sandwiched) a blood smear was then prepared, and iron particles smeared, taking into account the underlying hematite magnetic reach.

\section{4) Methodology: to Document the Lateral (Same Plane) and Vertical (Through A $1 \mathrm{Mm}$ Thick Glass) Magnetic Effect of Magnetite on Iron Particles in Fresh Blood Undergoing Coagulation.}

Relevant Note: Prior research had documented magnetic fields penetrating through glass barriers (8).

\section{Magnetized Magnetite Fragments in Fresh Blood and Color Changes}

When a permanently magnetized magnetite fragment was placed on a fresh blood smear, besides the RBCs attractions, shifting in the light spectrum ensued.

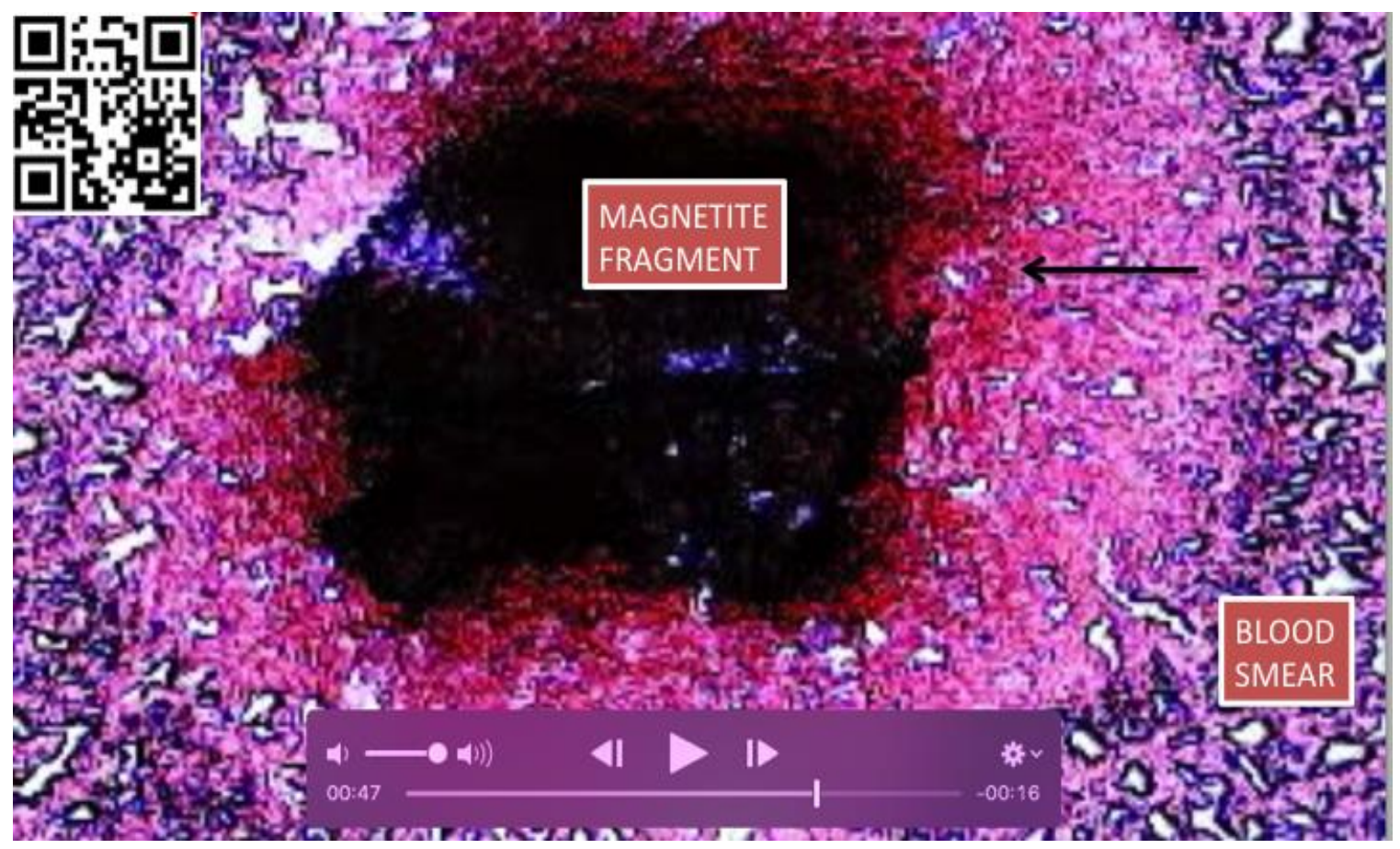

Figure 3: Frame 00:47" of video recording. Magnetite fragment placed directly on a fresh blood smear. Unedited image showing shift in white light spectrum towards light to dark pink during water evaporation. Suggested to scan QR Code in top right corner of image; and move rapidly the video playback cursor.

https://youtu.be/3wOX9AH2Yt8 or Scan QR Code in left open corner of image. 


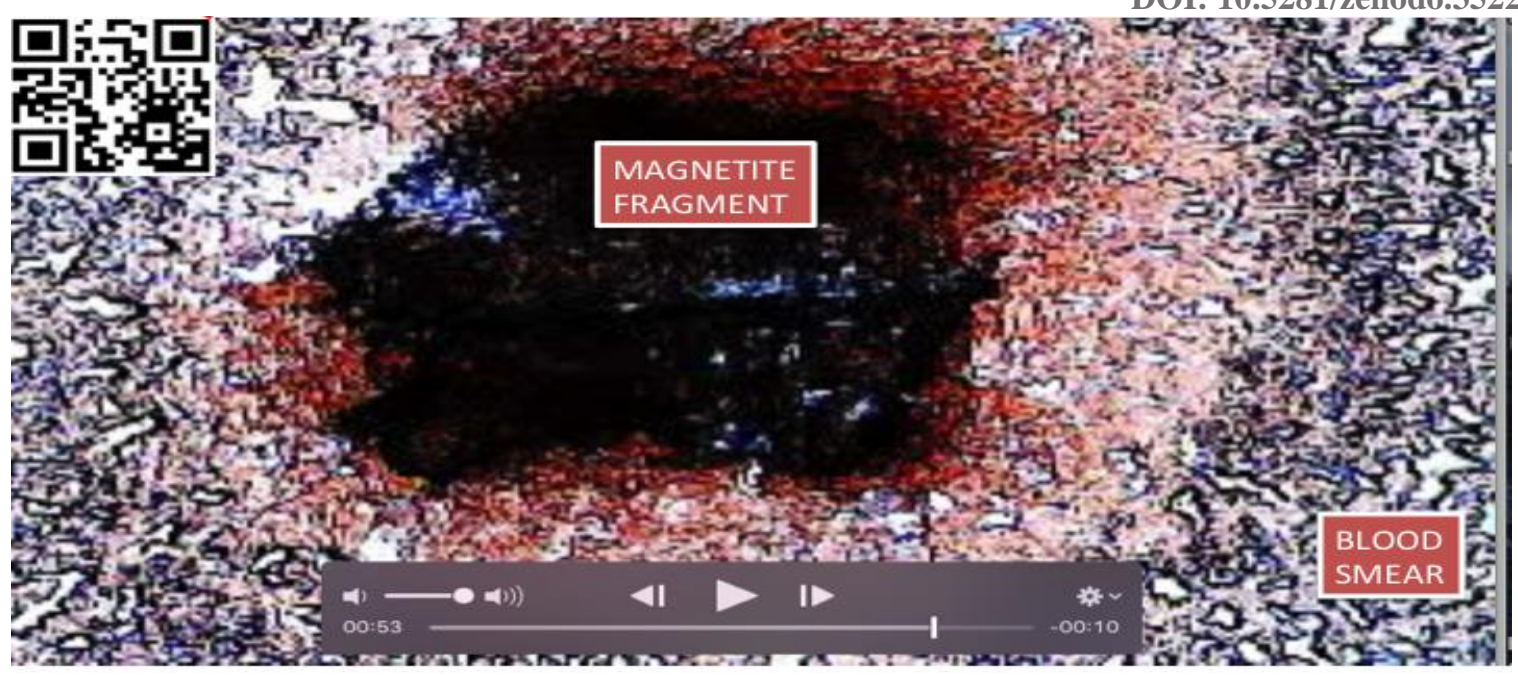

Figure 3A: Frame 00:53" of same video recording in Fig. 3 above. This time the light spectrum has shifted towards white. Some remanence of residual purple hue seen inside magnetite fragment.

https://youtu.be/3wOX9AH2Yt8 or Scan QR Code in left upper corner of image.

\section{Iron Filings in Fresh Bood Smear and Color Changes}

Paramagnetic metal iron particles are attracted to an external magnetic field. The figure and video recording below demonstrate changes in the light radiation spectrum triggered by the blood coagulation cascade. This could be described as a biophysical phenomenon, which is now possible to display for the first time due to a novel technique temporarily preserving fresh human blood properties on a glass smear a.k.a. TIBS. (Acronym for “Temporary In Vivo Blood Smear").
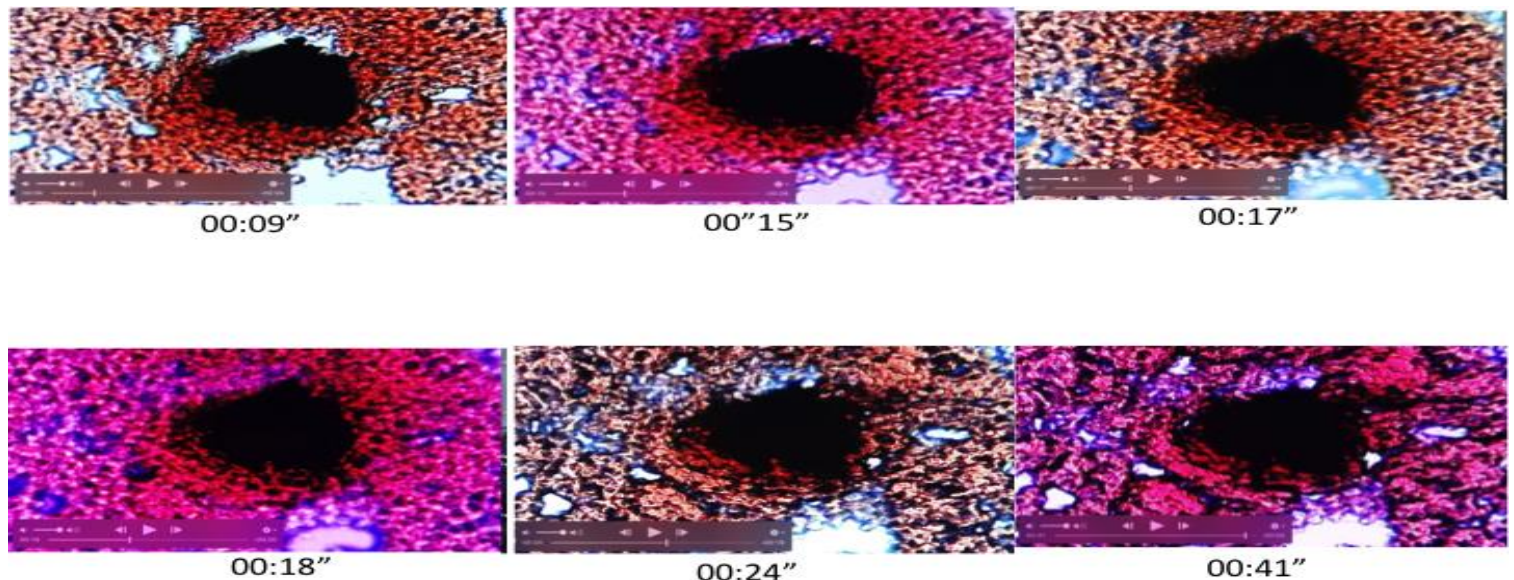

$00: 24 "$

PARAMAGNETIC METAL IRON FILING IN FRESH BLOOD SMEAR UNDERGOING COAGULATION

Figure 3B: Selected video frames showing changes in optical microscopy light spectrum detected during a coagulation cascade. Selected frames from video recording of metal iron filing placed on fresh blood smear. The iron filings are paramagnetic and known as "attracted to a magnetic field”. For details link to: https://youtu.be/cuwJeS5alT4 Or Scan QR Code in lower right corner. 
Iron Filing in Fresh Blood Smear Within Magnetite Magnetic Reach Range

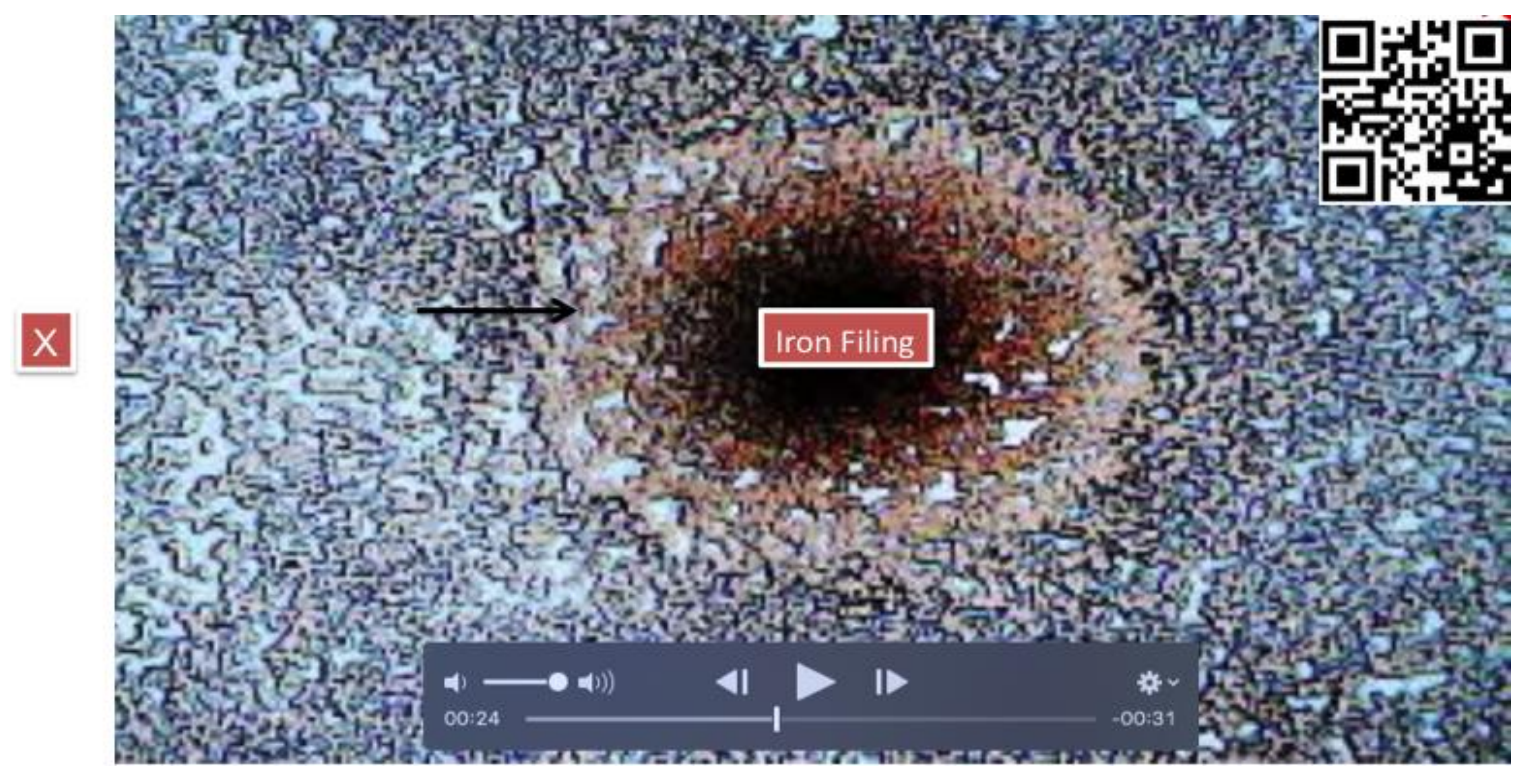

Figure 4: Iron filing on fresh blood under magnetite magnetic reach effect. Black Arrow= light radiation aura surrounding metal filing.

$\mathrm{X}=$ Magnetite Fragment outside viewing field (not shown). Compare with Fig. 2. Please visit link: https://youtu.be/tIcKeACe5dI

Or scan QR Code in upper right side of image.

\section{Iron Filing in Fresh Blood Smear In Magnetite Magnetic Reach Range Penetrating A 1 Mm Glass Slide}

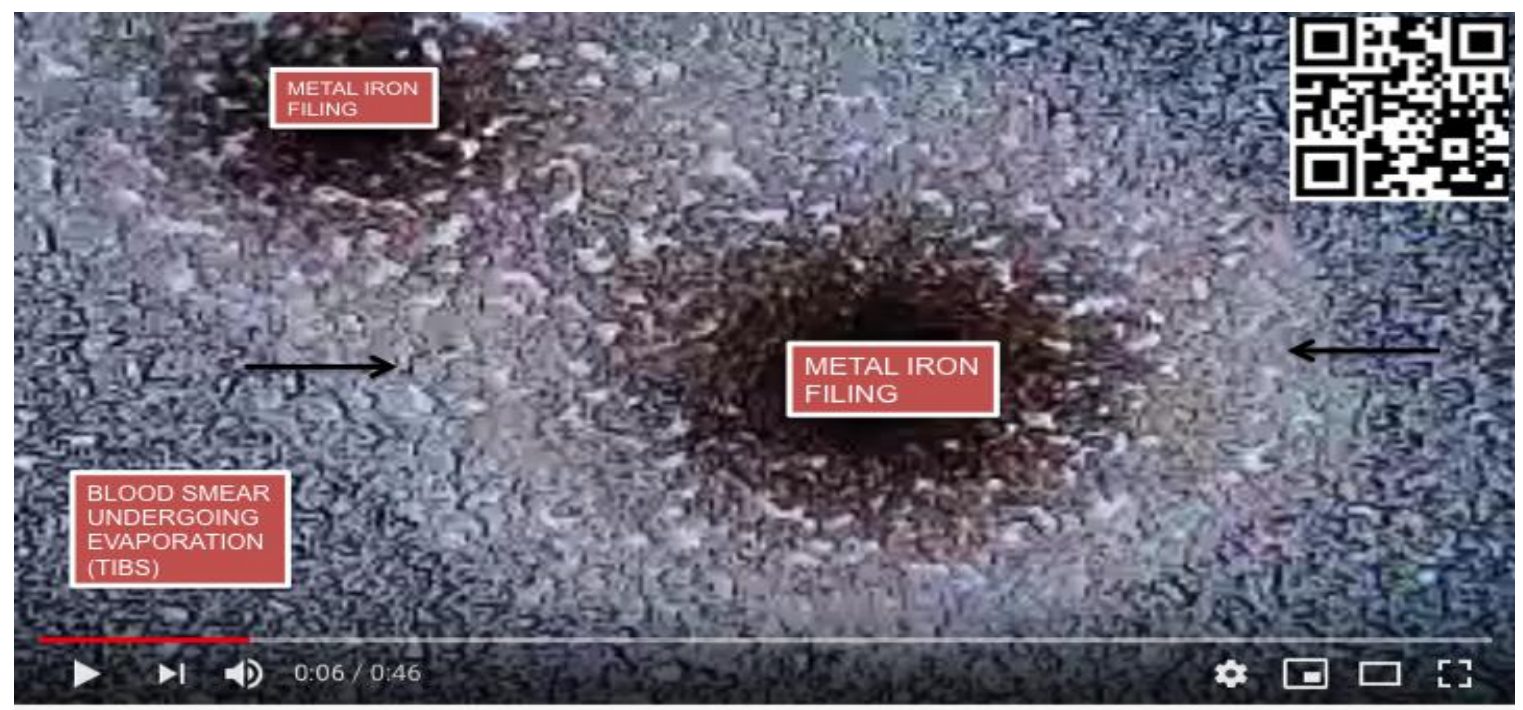

Figure 4A: Depicting magnetite fragment effect (through $1 \mathrm{~mm}$ glass slide) on metal iron particles placed on fresh blood smear. Black arrows pointing at induced light radiation from magnetite.

For additional details please link to https://youtu.be/zxBrCXCvEck or scan QR Code o top right corner of image. 


\section{Removal of Magnetite Fragment Post Water Evaporation Showing Hidden Foreign Material} Blood Attraction

Similar to deformed RBCs caused by iron filings (Fig. 2A), the removal of the magnetite fragment post water evaporation also showed deformation of RBCs. The next three images document the above statement.

Note: In the spectrum of particles evaporation on fresh blood smears, the larger the particle, the less noticeable is the RBC attraction. A hidden attraction is likely to occur under the large particle causing the RBCs deformation. This was documented in two additional different experiments (not shown).

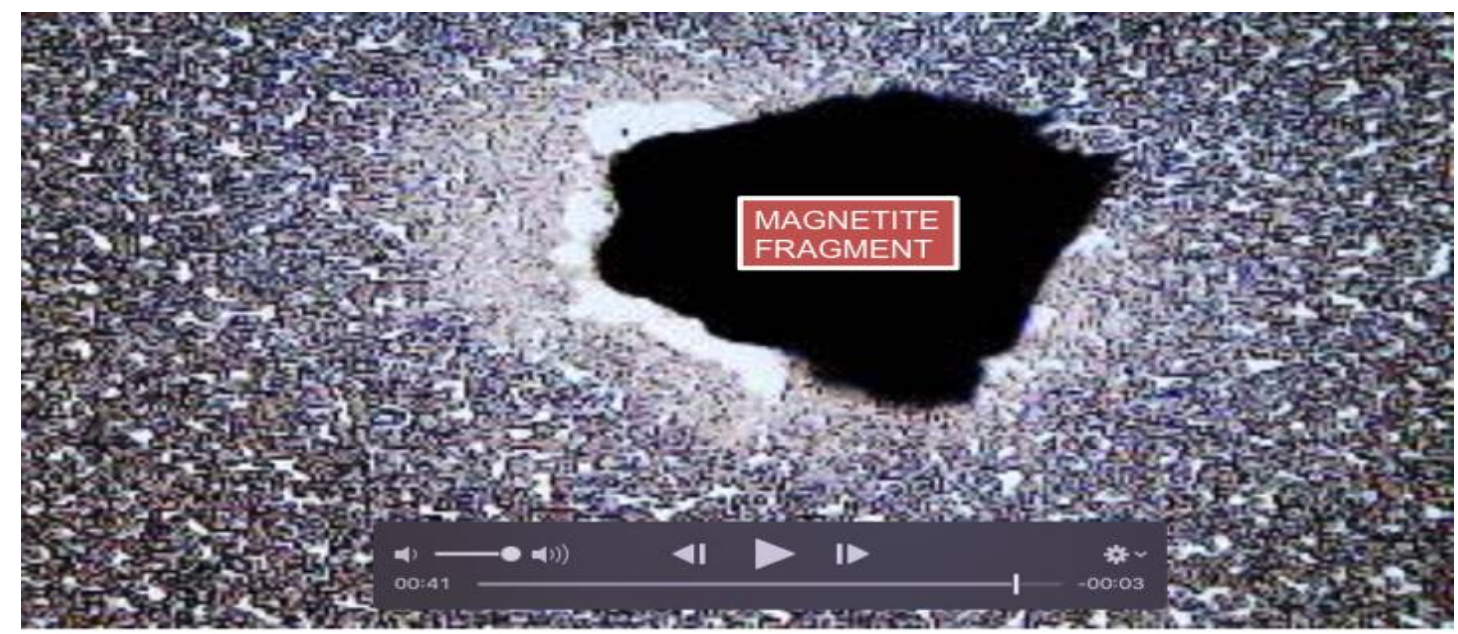

Figure 5: Small Magnetite fragment placed on fresh blood smear. - Image at end of water evaporation. Compared with iron filing on fresh blood smear, there is an apparent absence of RBC motion.

For additional details link to https://youtu.be/54BaJzNhuJc Or Scan QR Code.

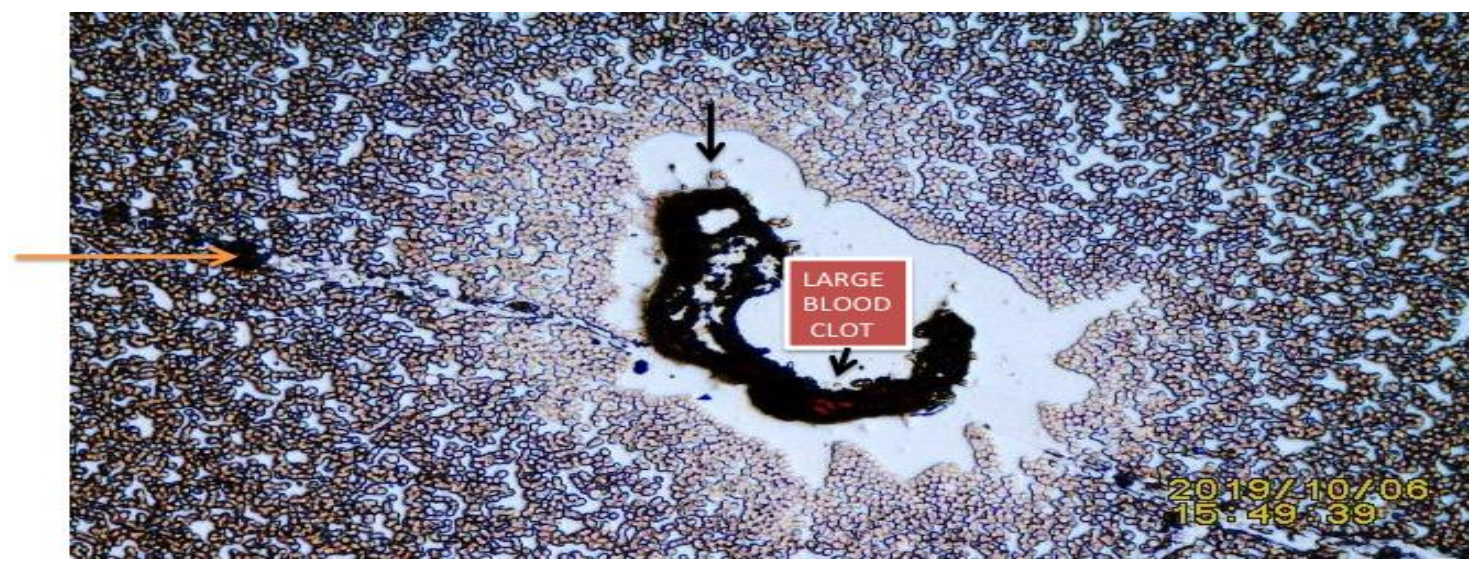

Figure 5A: Small magnetite fragment physically removed showing material adhered to slide.

Large Red Arrow= Magnetite fragment removal trail by wooden toothpick. Top Black Arrow= Compressed deformed RBCs image. Bottom Black Arrow= Large Blood Clot. 


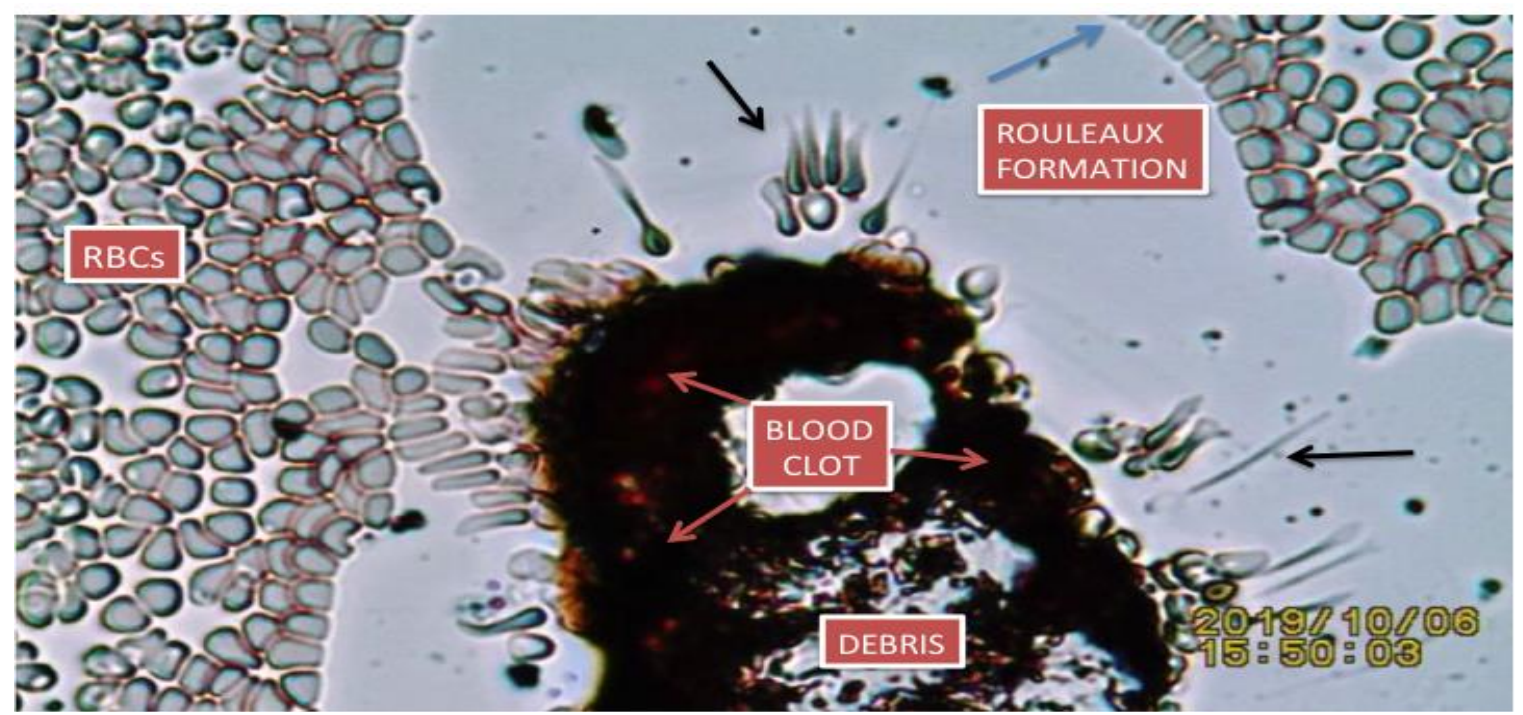

Figure 5B: Enlarged video frame from Fig. 5A. Showing: Black Arrows= Deformed RBCs. Red Arrows $=$ Blood Clot. Purple Arrow= Rouleaux Formation. Please compare similarities with Fig. 2A (Effect of Iron particles)

\section{Discussion}

As stated, the purpose of this manuscript is to expand previous research findings where a change in light radiation intensity was observed during blood coagulation. Please view Exhibit II below depicting the intrinsic color changes of the visible light spectrum as perceived by the human eye. As the temperature rises (energy), the spectrum shifts towards shorter wavelengths or blue (Ehibit II) below:

COLD

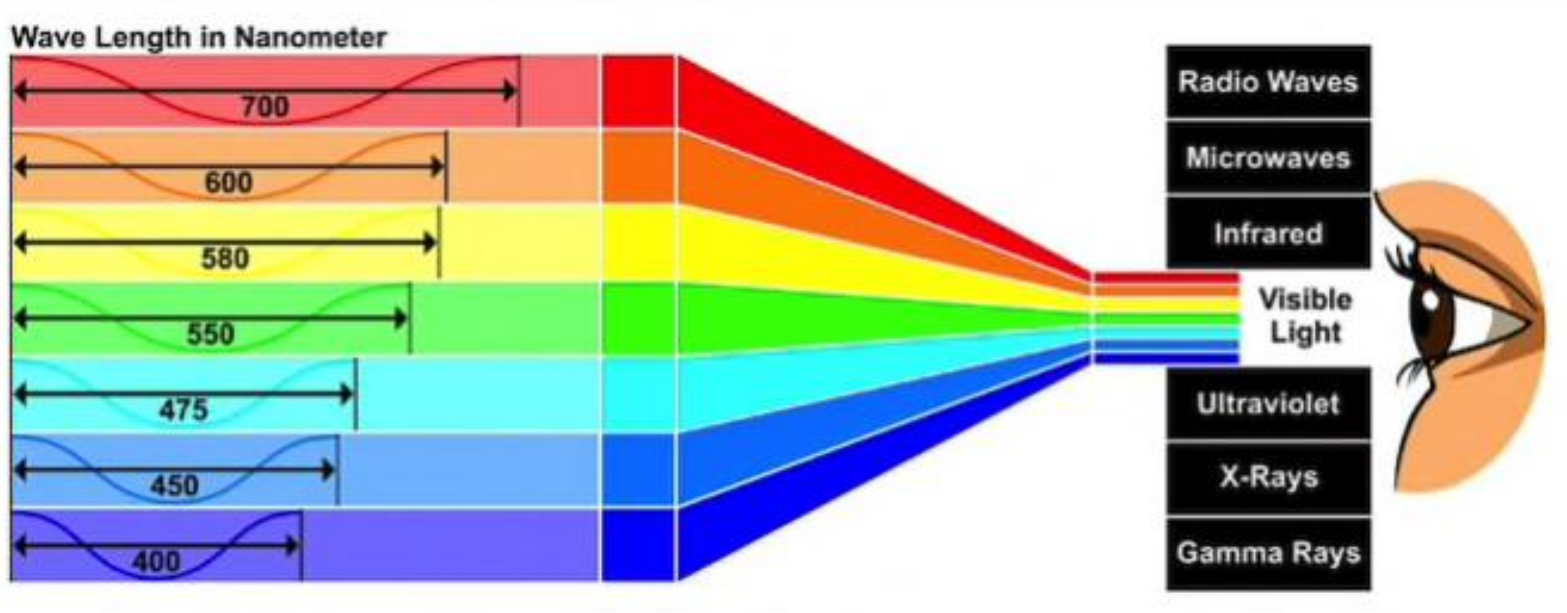

HOT

\section{EXHIBIT II}

Exhibit showing the visible light radiation spectrum by the human eye Image licensed for downloading from Shutterstock,com 


\section{Relationship of Visible Wave Length and Energy}

Since the energy of a wave is directly proportional to its frequency, but inversely proportional to its wavelength, then it could be inferred that some of the introduced experiments are in support of energy changes during blood coagulation. (Figs 3,3A,3B). In other words, the greater the energy, the larger the frequency and the shorter (smaller) the wavelength. As shown in Exhibit I above, the shorter the visible electromagnetic wavelength (blue) the higher the energy.

\section{Proposed Utility of the Fresh Blood Smear Technique}

One possible utility of the technique herein introduced is to allow researchers energy displays in color images during the blood coagulation cascade. How would the presence of blood disorders, such as sickle cell disease, leukemias, inherited hemophilia or others differ in energy displays?

\section{Conclusions}

\section{New Findings and Recommendations from this Research.}

\section{Findings}

- Prior research hypothesized a magnetic cross-talk between Red Blood Cells (RBCs) and foreign material triggering RBCs deformation in the form of teardrops (Figs 2A,5B). The new data now points at an additional factor, such as hydrodynamic physical effect such as the RBCs sudden migration and adherence to magnetite and iron filings. The Interfacial Adsorption Principle (9) and the contact activation of blood plasma coagulation as factors (10) as underlying mechanisms (Fig 2)

- Magnetism was documented causing changes in the visual light spectrum (energy fluctuations) during blood coagulation (Figs 3,3A,4,4A)

- Since the energy of a wave is inversely proportional to its wavelength (color changes), then it could be inferred that some of the introduced experiments are in support of energy changes during blood coagulation. (Figs 3,3A,3B).

- Magnetite fragments intrinsic magnetism causes energy fluctuations during the blood coagulation cascade

- Paramagnetic iron filings also cause energy fluctuations during the blood coagulation cascade. Since paramagnetic material (iron filings) have a high susceptibility towards an external magnetic field; is possible that energy fluctuations during blood coagulation (or the intrinsic blood magnetic profile) could interact with iron particles.

- The herein reported findings are also in support of a previously published hypothesis on cancer genesis; as quoted "The hypothesis can be summarized as follows "One additional cause of the origin of cancer is directly related to exogenous and/or endogenous EMFs damaging the DNA within the eukaryotic cell" (11).

\section{Recommendations}

Due to its simplicity, the methodology herein introduced could potentially aid in research correlating blood coagulation energy changes and other blood diseases. Of major importance is also the idea that this manuscript has introduced a mechanism for cancer genesis as supported by epidemiological studies correlating air pollution and cancer. $(12,13,14)$. 


\section{Definitions of Terms}

Bioactive materials: A bioactive material is one that elicits a specific biological response at the interface of the material, which results in the formation of a bond between the tissues and the material, shown first in 1969.

Biophysics: Is an interdisciplinary science that applies approaches and methods traditionally used in physics to study biological phenomena.

Interfacial Adsorption: Mechanism responsible for foreign material as a factor in the clotting blood. First described by Joplin JM. In 1928.

Magnetite: Commonly known as Lodestone, this magnetic form of Magnetite is the only mineral that is a natural magnet.

Paramagnetic Materials: Ferromagnetic materials (such as metal iron filings) have a large, positive susceptibility to an external magnetic field.

Superparamagnetism: Superparamagnetism is the magnetic behavior associated with magnetic nanoparticles, generally of ferromagnetic or ferrimagnetic materials.

(TIBS): Acronym for "Temporary In Vivo Blood Smears". Quoted for clarity in communication as "Fresh Blood Smears" in this manuscript. First described by Embí AA in 2018.

\section{References}

[1] C. K. N. Patel and A. C. Tam, “Optical absorption coefficients of water,” Nature London 1979 280, 302-304.

[2] Embi AA. (2019). "ENERGY DETECTION IN THE FORM OF LIGHT RADIATION AT END OF HUMAN BLOOD COAGULATION CASCADE- THE OPTICAL ABSORPTION OF WATER VS. FIBRIN BURST ENERGY RELEASE." International Journal of Research Granthaalayah, 7(9), 200-212. https://doi.org/10.5281/zenodo.3472760. XXX

[3] Embi AA. "BIOMAGNETISM AS FACTOR IN RED BLOOD CELLS DEFORMATION." International Journal of Research - Granthaalayah, $6 \quad$ (12), 46-57. 2018 https://doi.org/10.5281/zenodo.2528404.

[4] Embi AA. Hair and blood endogenous low level biomagnetic fields cross-talk effects on fibrin inhibition and rouleaux formation. IJGR, 2018 6(11), 200-208. https://doi.org/10.5281/zenodo. 1845985.

[5] Embi AA. Expanding the role of magnetic fields in red blood cells deformations: Demonstration of paramagnetic and diamagnetic fields. IJRG, 2019 7(2), 214-220. https://doi.org/10.5281/zenodo.2587463

[6] Shabanova, E.M., Drozdov, A.S., Fakhardo, A.F., Dudanov, I.P., Kovalchuk, M., \& Vinogradov, V.V. (2017). Thrombin@Fe3O4 nanoparticles for use as a hemostatic agent in internal bleeding. Scientific Reports.Sci Rep. 2018 Jan 10;8(1):233. doi: 10.1038/s41598-017-18665-4.

[7] Barbara Maher, Imad AM, AhmedVassil KarKarloukovski. Magnetite pollution nanoparticles in the human brain Proceedings of the National Academy of Sciences 2016 113(39) DOI: 10.1073/pnas. 1605941113

[8] Abraham A. Embi, Benjamin J. Scherlag Demonstration of Human Hair Follicle Biomagnetic Penetration Through Glass Barriers International Journal of Materials Chemistry and Physics Vol. 2, No. 2, 2016, pp. 71-74 http://www.aiscience.org/journal/ijmcp

[9] Johlin JM. Interfacial Adsorption as a Factor in the Clotting of Blood Plasma. J Biol Chem. 1929; 81:99-113.

[10] Vogler, E. A., \& Siedlecki, C. A. Contact activation of blood-plasma coagulation. Biomaterials, 30(10), 1857-1869. doi:10.1016/j.biomaterials.2008.12.041 
[11] Embi A. A. (2016). Endogenous electromagnetic forces emissions during cell respiration as additional factor in cancer origin. Cancer cell international, 16, 60. doi:10.1186/s12935-016-0337$\mathrm{y}$

[12] Pedersen, D.U., Durant, J.L., Taghizadeh, K., Hemond, H.F., Lafleur, A.L., \& Cass, G.R. Human cell mutagens in respirable airborne particles from the northeastern United States. 2. Quantification of mutagens and other organic compounds. 2005 Environmental science \& technology, 3924 , 9547-60.

[13] Consonni, D., Carugno, M., De Matteis, S., Nordio, F., Randi, G., Bazzano, M., ... Landi, M. T. Outdoor particulate matter (PM10) exposure and lung cancer risk in the EAGLE study. 2018 PloS one, 13(9), e0203539. doi:10.1371/journal.pone.0203539

[14] Kyoung Jin Kim, Jinyoung Shin and Jaekyung Choi. Cancer Risk from Exposure to Particulate Matter and Ozone According to Obesity and Health-Related Behaviors: A Nationwide PopulationBased Cross-Sectional Study. Cancer Epidemiol Biomarkers Prev February 12019 (28) (2) 357362; DOI: 10.1158/1055-9965.EPI-18-0508

\footnotetext{
*Corresponding author.

E-mail address: embi21@ att.net
} 